\title{
Kinetic theory for transverse opto-mechanical instabilities
}

\author{
E. Tesio, G.R.M. Robb, T. Ackemann, W.J. Firth, and G.-L. Oppo \\ SUPA and Department of Physics, University of Strathclyde, Glasgow G4 ONG, Scotland, UK
}

\begin{abstract}
We investigate transverse symmetry-breaking instabilities emerging from the opto-mechanical coupling between light and the translational degrees of freedom of a collisionless, damping-free gas of cold, two-level atoms. We develop a kinetic theory which can also be mapped on to the case of an electron plasma under ponderomotive forces. A general criterion for the existence and spatial scale of transverse instabilities is identified; in particular, we demonstrate that monotonously decreasing velocity distribution functions are always unstable.
\end{abstract}

PACS numbers: 05.65.+b, 42.65.Sf, 37.10.Vz

The spontaneous emergence of ordered states from homogeneous initial conditions is a preeminent feature of nonlinear systems driven far from thermodynamic equilibrium. Since the pioneering work by Turing [1], it became clear that a variety of nonlinear physical systems can spontaneously break spatio-temporal symmetry as the result of instabilities to infinitesimal perturbations. Typically, perturbations at a given spatial and/or temporal scale become unstable when the amount of injected energy exceeds a threshold value. Such a spontaneous self-organization manifests itself in many branches of physics: plasma instabilities, for instance, play a major role in fusion research [2]. Symmetry breaking instabilities are ubiquitous in chemistry, fluid dynamics, or biology [3]. In optical systems, optical nonlinearities have been shown to lead to self-organization in the plane transverse to the light propagation, in a variety of media and geometrical configurations [4-8].

In recent years, self-organizing instabilities due to the opto-mechanical coupling of light and cold [9-14] and ultracold $[15,16]$ atoms have attracted remarkable interest. In many of these schemes a pump beam is scattered by the gas into an externally imposed mode (often selected by a cavity). The interference between this mode and the pump then provides a modulated light pattern, which via dipole forces leads to a spatial rearrangement of atoms. The emerging density gratings resulting from the interference of this mode and the pump then provide positive feedback by scattering photons into the self-sustained mode. In these arrangements, the spatial scale of the emerging structure is predetermined by the light wavelength and the geometrical configuration. Multi-mode cavity setups displaying continuous symmetry breaking and spin-glass behavior in ultracold gases also attracted remarkable interest [17]. Alternative and naturally multimode schemes are possible in cold atoms, where spatial organization emerges in the plane transverse to the propagation of a single beam, with self-selected scales. It is in fact expected that atomic transport due to dipole forces can lead to nonlinear effects in cold atoms analogous to the Kerr effect in the hot-atoms case [18, 19]. Self-organization in a counterpropagating geometry was first analyzed in [20] and some experimental evidence for its existence was found in [21]. In [22] it was shown that opto-mechanical forces in a ring cavity configuration can lead to an instability even in the absence of any optical (Kerr) nonlinearity, thus providing a new patternforming mechanism on their own. The study of transverse opto-mechanical instabilities in cold atoms has been so far limited to the case where strong velocity damping is provided by optical molasses $[20,22]$. Recent experiments in cold Rb have however shown spontaneous symmetry breaking due to opto-mechanical coupling, but in the absence of such damping [23]. The need of a satisfactory understanding of these results has thus been a strong motivation to the theoretical study of the damping-free case by extending the analysis of [20] and [22].

Nonlinear effects of conceptually similar origin have also been demonstrated in plasmas, such as self-focusing and filamentation [24-26]. Here the nonlinearity originates from ponderomotive forces acting on the plasma and pushing the electrons away from high-intensity regions. In the presence of feedback this is expected to lead to a coupled light-density transverse self-organization, whose theoretical or experimental evidence is to our knowledge still lacking in the context of plasma physics. We also emphasize that the connection between plasmas and cold atoms can be extended beyond the correspondence between dipole and ponderomotive forces. In fact, attractive (shadow) and repulsive (radiation pressure) forces exist inside magneto-optically trapped samples, which introduce an effective charge between the atoms and thus simulate electrostatic interaction [27-30]. Beside the fundamental interest in cold-atom instabilities, therefore, cold and ultracold atomic samples can also provide a powerful and highly controllable tool for the study of various plasma systems including quantum plasmas.

We consider a thermal gas of two-level atoms described by the distribution function $f(\mathbf{x}, \mathbf{v}, t)$. In what follows, $\mathbf{x}$ and $\mathbf{v}$ denote the positions and velocities in the plane transverse to the propagation of light, see Fig. 1. The gas is initially prepared at temperature $T$ and interacts with an optical beam at frequency $\omega_{0}$, tuned far from any 


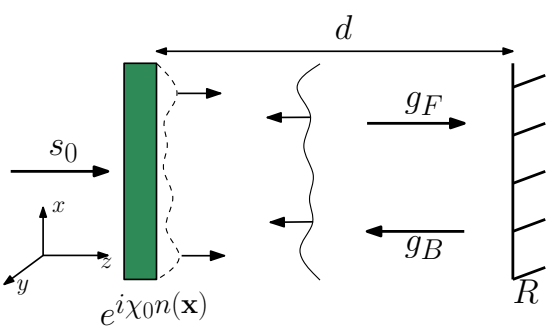

FIG. 1: Sketch of the single mirror feedback scheme. A plane wave of intensity $s_{0}$ interacts with a sample of two-level, cold atoms. The gas (or plasma) imposes a phase shift $\chi_{0} n(\mathbf{x}, t)$ on the field, where $n$ is the atomic density. Transverse fluctuations in the phase profile of the forward field $g_{F}$ (dashed line) are converted into amplitude modulations for the backward field $g_{B}$ (full line) by the free-space propagation to the mirror (reflectivity $R$, distance $d$ ) and back.

atomic transition. Due to this large detuning assumption, heating effects are negligible. At the low temperatures obtainable by laser cooling $(T \sim 100 \mu \mathrm{K})$, collisions are also negligible and the dynamics is governed by the collisionless Boltzmann equation

$$
\frac{\partial f}{\partial t}+\mathbf{v} \cdot \frac{\partial f}{\partial \mathbf{x}}+\frac{\mathbf{F}}{M} \cdot \frac{\partial f}{\partial \mathbf{v}}=0
$$

where $M$ is the atomic mass, and $\mathbf{F}$ the force acting on the gas. In cold atoms this is given by the dipole force $\mathbf{F}=-\frac{1}{2} \hbar \delta \nabla_{\mathbf{x}} \log [1+s(\mathbf{x})]$, where $\delta=\omega_{0}-\omega_{\text {at }}$ is the atom-light detuning and $s(\mathbf{x})=I(\mathbf{x})\left[I_{\text {sat }}\left(1+4 \delta^{2} / \Gamma^{2}\right)\right]^{-1}$ the saturation parameter associated with the total intensity $I(\mathbf{x})$ illuminating the gas. Scattering forces are neglected by assuming the detuning to be much larger than the atomic linewidth, $|\delta| \gg \Gamma$.

The plasma case is retrieved by identifying $f$ as the electron distribution, $M=m_{\mathrm{e}}$ as the electron mass and $\mathbf{F}_{\mathrm{pm}}=-\left[e^{2} /\left(2 \varepsilon_{0} c m_{\mathrm{e}} \omega_{0}^{2}\right)\right] \nabla_{\mathbf{x}} I(\mathbf{x})$ as the ponderomotive force, with $e$ the electron charge, $\varepsilon_{0}$ the vacuum permittivity and $c$ the speed of light in vacuum.

To analyze the stability of the perturbations, we Fouriertransform our quantities in space and Laplace-transform in time. This leads to the usual relations $\partial_{\mathbf{x}} \rightarrow i \mathbf{q}$ and $\partial_{t} \rightarrow-i \omega$, with growing perturbations identified by $\operatorname{Im}(\omega)>0$. Linearization of Eq. (1) then leads to

$$
(-i \omega+i \mathbf{q} \cdot \mathbf{v}) f_{1}=i \frac{\hbar \delta}{2 M} \frac{s_{1}(\mathbf{q}, \omega)}{1+s_{\mathrm{h}}} \mathbf{q} \cdot \frac{\partial f_{0}}{\partial \mathbf{v}}+f_{1}(0),
$$

where $f_{1}(0)$ denotes the initial disturbance, and we took into account the fact that the force is a first order quantity involving spatial gradients. Scaling is such that $s_{0}=\left|g_{F}\right|^{2}$ represents the saturation parameter associated to the forward field, and $s_{\mathrm{h}}=(1+R) s_{0}$ is the homogeneous solution ( $R$ being the mirror transmittivity, see Fig. 1). Since we are interested in transverse effects only, we average over the longitudinal degrees of freedom and take the total intensity as $s=s_{0}+\left|g_{B}\right|^{2}$. The
Laplace transform ensures that causality is preserved by correctly viewing Eq. (1) as an initial value problem. To obtain $s_{1}(\mathbf{q}, \omega)$ we first calculate the forward field $g_{F}$ at the exit of the medium. In the limit of small saturation parameters and large detuning we can neglect absorption and approximate the atoms as linear (Rayleigh) scatterers. The forward field will thus be phase-shifted as $g_{F} \rightarrow g_{F} \exp \left\{i \chi_{0} n(\mathbf{x}, t)\right\}$, where $\chi_{0}$ is the linear phase shift imposed by the cloud and $n$ the spatial density of the gas, obtained by integrating $f(\mathbf{x}, \mathbf{v}, t)$ over the entire velocity space. For a sample of two-level atoms with optical density $b_{0}$ one has $\chi_{0}=b_{0} \delta\left[\Gamma\left(1+4 \delta^{2} / \Gamma^{2}\right)\right]^{-1}$. Similarly, a plasma acts as a purely dispersive medium with a density-dependent susceptibility [2]. In what follows definitions are chosen so that the uniform density solution is $n_{\mathrm{h}}=1$. We then propagate the field to the mirror (distance $d$ ) and back to obtain the backward field $g_{B}$. If the backward field is perturbed as $g_{B}=g_{B}^{(0)}\left(1+b_{1}(\mathbf{x}, t)\right)$, the intensity perturbation $s_{1}(\mathbf{q}, \omega)=R s_{0}\left(b_{1}(\mathbf{q}, \omega)+c . c.\right)$ will depend on the gas distribution as:

$$
s_{1}(\mathbf{q}, \omega)=-2 R s_{0} \chi_{0} \sin \Theta_{q} \int d \mathbf{v} f_{1}(\mathbf{q}, \mathbf{v}, \omega),
$$

where $\Theta_{q}=\left(d / k_{0}\right) q^{2}$ is the diffractive phase shift, with $k_{0}$ the light wavenumber and $q=|\mathbf{q}|$ the transverse wavenumber. The key point of the single mirror feedback scheme is the conversion of phase perturbations into amplitude perturbations operated by the free-space propagation [5]. In fact, as phase fluctuations are converted into amplitude perturbations for the backward field, dipole forces are induced into Eq. (1). These in turns affect $n$ through the opto-mechanical coupling and consequently feed back to the backward field amplitude profile. If positive feedback can be obtained for a disturbance at some wavevector $\mathbf{q}$, an instability at that wavevector is expected.

To progress in the stability analysis we now seek a closed expression for $s_{1}$. Obtaining $f_{1}$ from Eq. (2) and using this result into (3) we reach the following expression for the intensity perturbation:

$$
\begin{aligned}
s_{1}(\mathbf{q}, \omega)=-2 R s_{0} \chi_{0} & \sin \Theta_{q} \int d \mathbf{v} \frac{f_{1}(0)}{i \mathbf{q} \cdot \mathbf{v}-i \omega} \times \\
\times & {\left[1+K_{q} \int d \mathbf{v} \frac{\hat{e}_{\mathbf{q}} \cdot \partial f_{0} / \partial \mathbf{v}}{\hat{e}_{\mathbf{q}} \cdot \mathbf{v}-\omega / q}\right]^{-1}, }
\end{aligned}
$$

where $\hat{e}_{\mathbf{q}}$ is the unit vector oriented as $\mathbf{q}$ and we defined

$$
K_{q}=\frac{\hbar \delta}{M} \frac{R s_{0}}{1+(1+R) s_{0}} \chi_{0} \sin \Theta_{q} .
$$

The behavior of $s_{1}(\mathbf{x}, t)$, and thus the dynamics of the system, is fully determined by the inverse Fourier and Laplace transforms of $s_{1}(\mathbf{q}, \omega)$. Under regularity assumptions for $f_{1}(0)$ and $\partial f_{0} / \partial \mathbf{v}$, the integrands above have no singularities and the only contributions to the inverse 
Laplace transforms are given by the zeros of a 'dielectric function':

$$
D(\mathbf{q}, \omega)=1+K_{q} \int d \mathbf{v} \frac{\hat{e}_{\mathbf{q}} \cdot \partial f_{0} / \partial \mathbf{v}}{\hat{e}_{\mathbf{q}} \cdot \mathbf{v}-\omega / q}=0 .
$$

A formally identical result is obtained for the case of an electron plasma under the action of ponderomotive forces. The plasma case is retrieved by substituting $K_{q}$ with $K_{q}^{\mathrm{PL}}=\left[e^{2} /\left(\varepsilon_{0} c m_{\mathrm{e}}^{2} \omega_{0}^{2}\right)\right] R I_{0} \chi_{0} \sin \Theta_{q}$, with $I_{0}$ the non-rescaled incident intensity $\left(\right.$ in $\mathrm{Wm}^{-2}$ ).

A first general result can be found from Eq. (4) by expanding $\omega=\omega_{\mathrm{r}}+i \omega_{\mathrm{i}}$. Requiring both real and imaginary parts of $D(\mathbf{q}, \omega)$ to be zero leads to the condition:

$$
1+K_{q} \int d \mathbf{v} \frac{\left(\hat{e}_{\mathbf{q}} \cdot \partial f_{0} / \partial \mathbf{v}\right)\left(\hat{e}_{\mathbf{q}} \cdot \mathbf{v}\right)}{\left(\hat{e}_{q} \cdot \mathbf{v}-\omega_{\mathrm{r}} / q\right)^{2}+\left(\omega_{\mathrm{i}} / q\right)^{2}}=0 .
$$

For any monotonically decreasing function $f_{0}(|\mathbf{v}|)$, such as a Maxwellian, we find that an instability is in principle always possible since $\left(\hat{e}_{\mathbf{q}} \cdot \partial f_{0} / \partial \mathbf{v}\right)\left(\hat{e}_{\mathbf{q}} \cdot \mathbf{v}\right)<0$. Such monotonicity is thus a sufficient criterion for the occurrence of an instability, though it is not necessary: also non-monotonic distributions may in fact satisfy the condition (5). Eq. (5) also imposes a general restriction on the possible unstable wavenumbers in the single mirror arrangement. Since in two-level atoms $\chi_{0} \propto \delta^{-1}$ we have that $\delta \chi_{0}>0$ always, and an instability is found only in the regions where $\sin \Theta_{q}>0$. Similar considerations apply to the plasma case, where no red-detuning analog exists. One could see the opto-mechanical mechanism as responsible for a Kerr-like, self-focusing nonlinearity independent of the sign of the detuning. This was already recognized by Ashkin in early studies on dielectric particles [31], and remains true in the damped case of [22]. However, an important difference with soft-matter studies is that no velocity damping is present in our system. We remark that the result (5) is a general property of the opto-mechanical mechanism investigated here, and can be generalized to different geometries (e.g. a ring cavity). Eq. (5) should be contrasted to the NewcombGardner theorem in plasma physics [32], stating that plasma (Langmuir) waves are always stable if the initial velocity distribution is monotonically decreasing. For the case analyzed here of transverse perturbations and feedback, instead, we demonstrate an instability for monotonically decreasing $f_{0}$. We note that Eq. (5) identifies an instability both for dipole forces in cold atoms and pondermotive forces in plasmas.

Let us go back to Eq. (4), and focus for simplicity our attention to one transverse dimension. If $f_{0}(|v|)$ is taken as the Maxwellian $f_{0}=\left(2 \pi v_{\mathrm{th}}^{2}\right)^{-1 / 2} \exp \left(-\frac{1}{2} v^{2} / v_{\mathrm{th}}^{2}\right)$, where $v_{\mathrm{th}}^{2}=k_{B} T / M$, there is no analytic solution to the dispersion integral in Eq. (4). However, if we restrict ourselves to the threshold condition $\omega=0$ the dispersion integral simply reduces to the normalization condition for $f_{0}$ and

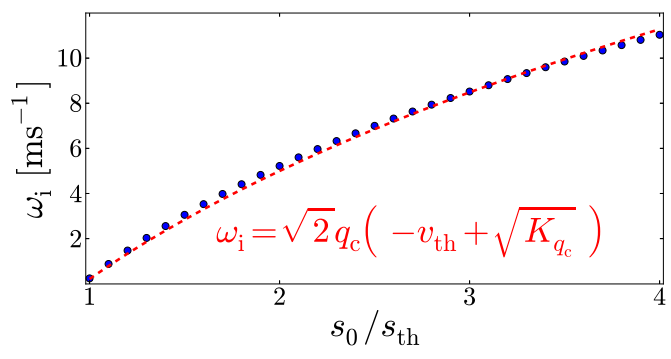

FIG. 2: (Color online) Linear growth rate at the critical wavenumber $q_{\mathrm{c}}=\sqrt{\pi k_{0} / 2 d}$. Dots are numerical evaluations of the dispersion relation (4) for the Maxwell distribution, the red line is the expression of Eq. (7). Parameters are: $\delta=30 \Gamma$, $\chi_{0}=1, R=1, d=5 \mathrm{~mm}$ and $T=300 \mu \mathrm{K}$.

we obtain a threshold condition for the injected saturation parameter:

$$
s_{0}=s_{\mathrm{th}} \equiv\left[\frac{\hbar \delta}{k_{B} T} R \chi_{0} \sin \Theta_{q}-(1+R)\right]^{-1} .
$$

The most unstable wavenumber (with minimum threshold) is given by the condition $\sin \Theta_{q}=1$, and a threshold is found also for the phase shift: $\chi_{0}>\left(\hbar \delta / k_{B} T\right)^{-1}(1+$ $R) / R$. Eq. (6) thus shows that an instability is possible for a Maxwellian gas and provides the threshold condition. We remark that the threshold (6) is identical to the one found in the presence of optical molasses, i.e. by adapting the model of [22] to the single mirror feedback geometry. However, Eq. (6) still leaves us without any information on the timescale of the process. Such information requires the calculation of the growth rate although, as stated earlier, no analytic solution is possible for the dispersion integral in (4). The dispersion integral can be analytically solved for the case of a Lorentzian velocity distribution $f_{0}=\pi^{-1} v_{\mathrm{th}} /\left[v^{2}+v_{\mathrm{th}}^{2}\right]$ : for this case the growth rate is purely imaginary, $\omega=i \omega_{\mathrm{i}}$, and given by

$$
\omega_{\mathrm{i}}=|q|\left\{-v_{\mathrm{th}}+\sqrt{K_{q}}\right\} .
$$

Since we do not expect the particular form of the distribution to be relevant in determining the qualitative features of the process, we numerically solve the Maxwellian dispersion relation $D\left(q, i \omega_{\mathrm{i}}\right)=0$ for a given $q$, and look for an equivalent expression of the growth rate. We choose here to investigate the critical wavenumber $q_{\mathrm{c}}=\sqrt{\pi k_{0} / 2 d}$ (which satisfies $\left.\sin \Theta_{q_{\mathrm{c}}}=1\right)$. Fig. 2 shows the growth rate $\omega_{\mathrm{i}}$ for the Maxwellian case for $\delta=30 \Gamma$, $\chi_{0}=1, R=1, d=5 \mathrm{~mm}$ and $T=300 \mu \mathrm{K}$. Taking the $D_{2}$ line of ${ }^{87} \mathrm{Rb}$ as a reference we use $\Gamma^{-1}=26 \mathrm{~ns}$ and $M=1.44 \times 10^{-25} \mathrm{~kg}$. A phase shift $\chi_{0}=1$ can be obtained by using a cloud with in-resonance optical density of 120 . We find that the growth rate is well described by

$$
\omega_{\mathrm{i}}=\sqrt{2} q_{\mathrm{c}}\left\{-v_{\mathrm{th}}+\sqrt{K_{q_{\mathrm{c}}}}\right\},
$$


which is identical, numerical prefactors aside, to the Lorentzian result. This result is easily extended to the plasma case by substituting $K_{q}$ with $K_{q}^{\mathrm{PL}}$ (see the discussion after Eq. (4)).

Fig. 3 shows the distribution function $f(x, v)$ obtained from numerical simulations of Eq. (1), for a final time of $3 \mathrm{~ms}$ and a transverse domain size of 5 critical wavelengths $\Lambda_{\mathrm{c}}=2 \pi / q_{\mathrm{c}}$. We drive the system with a pump beam of intensity $s_{0}=0.05$ (roughly 1.37 times the threshold) and observe the spontaneous emergence of a periodic optical potential, trapping a large fraction of the atoms in the intensity minima (maxima) for blue (red) detuning, respectively. We also show in Fig. 3 the intensity profiles $s(x)$ and the density distributions $n(x)$ obtained on the blue and red side of the resonance. The spatial scale of these structures is given by $\Lambda_{\mathrm{c}} \simeq 125 \mu \mathrm{m}$ with our choice of parameters, which is close to experimental observations [23].

We remark that the result (7) holds also for a different choice of the wavevector $q$, and that the growth rate depends only on the modulus $|q|$. The driving term $\sqrt{K_{q}}|q|$ directly depends from the intensity $s_{0}$, as well as the optical thickness of the medium and the light-atom detuning. On the other hand, a dephasing term $-v_{\text {th }}|q|$ originates from thermal motion. The balance of these two effects $\left(\omega_{\mathrm{i}}=0\right)$ leads to the threshold (6). Finally we note that the expression (6) implies that the instability threshold approaches zero as $T \rightarrow 0$ or, equivalently, as $f_{0}(\mathbf{v}) \rightarrow \delta(\mathbf{v})$ in our kinetic theory, which is a familiar result in the context of cold plasma instabilities. This is an important result since it gives considerable experimental flexibility in terms of achievable temperature and optical thickness. However, we note that decreasing the temperature below the Bose-Einstein condensation point the phenomenology is expected to change considerably. The study of transverse instabilities in a degenerate BoseEinstein condensate is beyond the scope of this Letter, and will be analyzed in future studies.

In conclusion, we have theoretically investigated optomechanical transverse instabilities in a single mirror feedback configuration. Similar results are expected for other configurations, such as counterpropagating beams and ring cavities. In contrast to previous studies in cold gases, no velocity damping is assumed in the system. Our kinetic theory for such a damping-free case is formally extendible to the case of a single-specie plasma, with the dispersion relation depending on the initial velocity distribution of the gas. We demonstrated that for monotonically decreasing velocity distributions of the gas a transverse instability due to opto-mechanical coupling is in principle always expected. For the case of a Maxwellian gas we identified the threshold condition for such an instability, together with an expression for the growth rate which agrees well with a numerical evaluation of the dispersion relation. A threshold appears when the driving
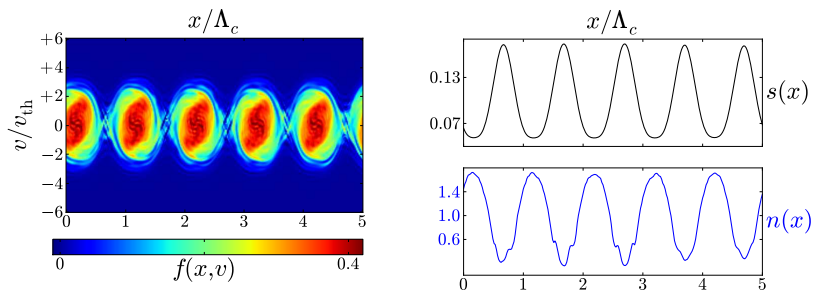

(a)
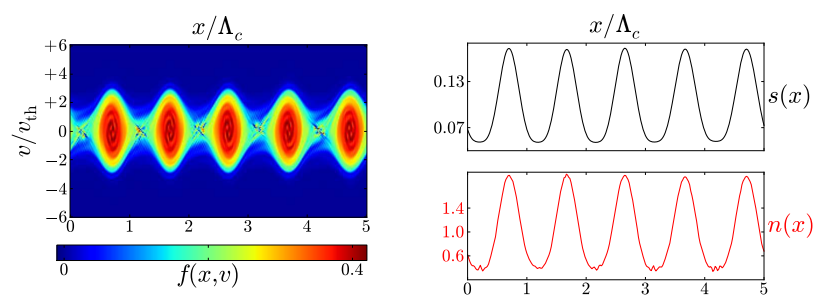

(b)

FIG. 3: Results from numerical simulations, for the same parameters as Fig. 2, injected intensity $s_{0}=0.05(\simeq 1.37$ times the threshold), and detuning $\delta=30 \Gamma$ (a) and $\delta=-30 \Gamma$ (b). For blue (red) detuning the maxima of the atomic density $n(x)$ align with the minima (maxima) of the optical intensity profile.

effect due to the pump and the dephasing effect due to thermal motion balance each other. The theory developed here does not take into account absorption or nonlinear dispersion originating from the internal structure of the atoms, so that the instability is entirely due to opto-mechanical coupling. The inclusion of such optical effects will be presented elsewhere, but we stress that they are not of principal importance in the large detuning limit. Future work could also extend the connection with plasma physics by including electrostatic-like effects [27-30], or investigate transverse instabilities in ultracold atomic gases and quantum plasmas.

We thank R. Kaiser and G. Labeyrie for fruitful discussions. Financial support from the Leverhulme Trust (Research Grant No. F/00273/0) and the EPSRC (for G.R.M.R., Grant No. EP/H049339) is gratefully acknowledged.

[1] A. M. Turing, Philosophical Transactions of the Royal Society of London 237, 37 (1952).

[2] F. F. Chen, Introduction to plasma physics and controlled fusion (Springer, 1984).

[3] M. C. Cross and H. S. Greenside, Pattern formation and dynamics in nonequilibrium systems (Cambridge University Press, 2009).

[4] G. Grynberg, E. Le Bihan, P. Verkerk, P. Simoneau, J. R. R. Leite, D. Bloch, S. Le Boiteux, and M. Ducloy, Opt. Comm. 67, 363 (1988).

[5] W. J. Firth, J. Mod. Opt. 37, 151 (1990). 
[6] L. A. Lugiato and R. Lefever, Phys. Rev. Lett. 58, 2209 (1987).

[7] R. Neubecker, G.-L. Oppo, B. Thuering, and T. Tschudi, Phys. Rev. A 52, 791 (1995).

[8] T. Ackemann and W. Lange, Phys. Rev. A 50, 4468(R) (1994).

[9] P. Domokos and H. Ritsch, Phys. Rev. Lett. 89, 253003 (2002).

[10] A. T. Black, H. W. Chan, and V. Vuletić, Phys. Rev. Lett. 91, 203001 (2003).

[11] R. Bonifacio and L. De Salvo, Nucl. Instrum. Methods Phys. Res. A 341, 360 (1994)

[12] S. Slama, S. Bux, G. Krenz, C. Zimmermann, and P. W. Courteille, Phys. Rev. Lett. 98, 053603 (2007).

[13] C. von Cube, S. Slama, D. Kruse, C. Zimmermann, P. W. Courteille, G. R. M. Robb, N. Piovella, and R. Bonifacio, Phys. Rev. Lett. 93, 083601 (2004).

[14] J. A. Greenberg and D. J. Gauthier, Eur. Phys. Lett. 98, 24001 (2012); Phys. Rev. A 86, 013823 (2012).

[15] K. Baumann, C. Guerlin, F. Brenneke, and T. Esslinger, Nature (London) 464, 1301 (2010).

[16] S. Inouye, A. Chikkatur, D. Stamper-Kurn, J. Stenger, D. Pritchard, and W. Ketterle, Science 285, 571 (1999).

[17] S. Gopalakrishnan, B. Lev, and P. Goldbart, Nat. Phys. 5, 845 (2009).

[18] M. Saffman, Phys. Rev. Lett. 81, 65 (1998).

[19] M. Saffman and Y. Wang, Lect. Notes Phys. 751, 361 (2008).
[20] M. Muradyan, Y. Wang, W. Williams, and M. Saffman, in Trends in Optics and Photonics (TOPS), vol. 80, Nonlinear Applications, OSA Technical Digest (OSA, Washington, D.C., 2005), paper ThB29.

[21] J. A. Greenberg, B. L. Schmittberger, and D. J. Gauthier, Opt. Expr. 19, 22535 (2011).

[22] E. Tesio, G. R. M. Robb, T. Ackemann, W. J. Firth, and G.-L. Oppo, Phys. Rev. A 86, 031801(R) (2012).

[23] G. Labeyrie, E. Tesio, P. M. Gomes, G.-L. Oppo, W. J. Firth, G. R. M. Robb, A. Arnold, R. Kaiser, and T. Ackemann (2013), to be published.

[24] C. Joshi, Phys. Scripta T30, 90 (1990).

[25] W. B. Mori, C. Joshi, J. M. Dawson, D. W. Forslund, and J. M. Kindel, Phys. Rev. Lett. 60, 1298 (1988).

[26] P. Kaw, G. Schmidt, and T. Wilcox, Phys. Fluids 16 , $1522(1973)$

[27] J. T. Mendonça, R. Kaiser, H. Terças, and J. Loureiro, Phys. Rev. A 78, 013408 (2008).

[28] J. T. Mendonça, Phys. Rev. A 81, 023421 (2010).

[29] H. Terças, J. T. Mendonça, and R. Kaiser, Eur. Phys. Lett. 89, 53001 (2010).

[30] J. T. Mendonça and R. Kaiser, Phys. Rev. Lett. 108, 033001 (2012).

[31] A. Ashkin, J. Dziedzic, and P. Smith, Opt. Lett. 7, 276 (1982).

[32] C. S. Gardner, Phys. Fluids 6, 839 (1963). 